Phase und nach den ersten 3 Monaten der offenen Phase erhoben.

Von den ursprünglich 132 an der Studie beteiligten Asthmatikern konnten von 128 die Lebensqualitätsdaten zu Studienbeginn und nach 12 Wochen erhoben werden, von 116 zusätzlich auch die Daten aus der offenen Phase. In der SF-36 berichteten die Asthmatiker zu Behandlungsbeginn erwartungsgemäß über eine signifikante Minderung ihrer Lebensqualität in Relation zur USDurchschnittsbevölkerung. Die Rücktitration der oralen Glukokortikoiddosen bei den Patienten unter den beiden
Dosierungen von Mometason ging mit einer im Vergleich zu Plazebo signifikanten $(p<0,05)$ Verbesserung in den Subskalen zur körperlichen Funktion und Leistungsfähigkeit einher. Auch in allen vier Subskalen der AQLQ-M (Atemlosigkeit, Stimmungsschwankungen, Störungen im Sozialleben und Besorgnisse um die Gesundheit) schnitten die mit Verum behandelten Patienten signifikant $(\mathrm{p}<0,05)$ besser ab als die Patienten unter Plazebo. In den 3 Monaten der offenen Studienphase stabilisierten oder verbesserten sich die Scores im SF-36 in den meisten Bereichen weiter.
Fazit: : Durch die zweimal tägliche Inhalation von Mometasonfuroat per Pulverinhalator kann bei Patienten mit schwerem Asthma die Gabe oraler Glukokortikoide reduziert oder sogar ganz eingestellt werden. Dies führt zu einer deutlichen Erhöhung der subjektiven Lebensqualität.

\section{$b k$}

Schmier J et al. Reduction in oral corticosteroid use with mometasone furoate dry powder inhaler improves healthrelated quality of life in patients with severe persistent asthma. J Asthma 2003; 40: 383-93

\title{
Operieren bei Asthma plus Reflux?
}

\section{Patienten mit Asthma leiden nicht selten auch unter gastroöso- phagealer Refluxkrankheit (GERD) und umgekehrt sind GERD- Patienten häufig auch von Asthma betroffen. Bisherige Studien deuten eine Besserung der Asthmasymptome durch eine Anti- Refluxbehandlung an. Dies wurde jetzt in einer Langzeitstudie überprüft.}

\begin{abstract}
Corscher aus Albuquerque, New Mexico, teilten 62 Patienten mit GERD plus Asthma randomisiert in drei Gruppen auf: 24 Patienten dienten als Kontrolle und nahmen bei Bedarf Antazida, 22 erhielten regelmäßig dreimal täglich den $\mathrm{H}_{2}$-Blocker Ranitidin und $16 \mathrm{~Pa}$ tienten wurden einer Fundiplikation unterzogen. Die Mindestbeobach-
\end{abstract} tungszeit betrug 2 Jahre. Asthma war in dieser Studie definiert als das Vorliegen einer entsprechenden Symptomatik und eines um mindestens 20\% verminderten $\mathrm{FEV}_{1}$-Wertes unter der Gabe von 205,5 Dosierungseinheiten Metacholin oder das Vorliegen eines 20\%igen $\mathrm{FEV}_{1}$-Anstiegs unter Bronchodilatatoren.

In der Gruppe mit der chirurgischen Intervention stellte sich unmittelbar nach der Operation eine deutliche Reduktion von Symptomen wie Husten, nächtliche Wheezing-Attacken und Dyspnoe ein, in den beiden anderen Gruppen wurden keine auffälligen Symptomveränderungen bemerkt. Nach 2 Jahren waren die Asthmasymptome in der Operationsgruppe bei 74,9\% verschwunden, unter Ranitidin bei 9,1\% und in der Kontrollgruppe bei 4,2\%. Umgekehrt hatte sich der Gesamtasthmastatus in der Kontrollgruppe bei $47,8 \%$, in der Ranitidin-

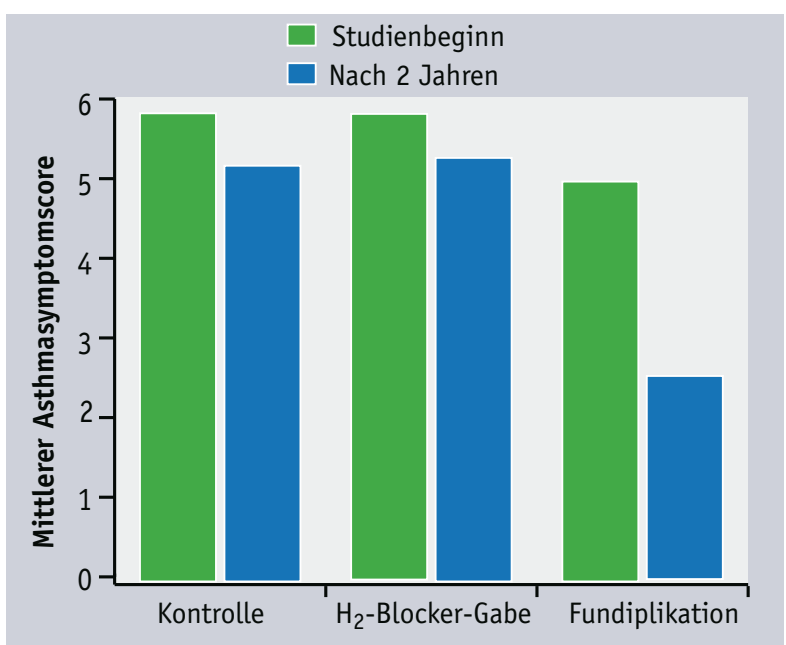

Veränderung des mittleren Asthmasymptomscore im Vergleich der drei Studiengruppen gruppe bei $36,4 \%$ und in der Operationsgruppe bei $12,5 \%$ verschlechtert.

Während sich der durchschnittliche Asthmasymptomscore nach der Fundiplikation mit $\mathrm{p}=0,0009$ hochsignifikant gegenüber den Kontrollgruppen besserte, war weder bei den Lungenfunktionsmessungen noch bei der benötigten Asthmamedikation ein Unterschied zwischen den Gruppen festzustellen.

Fazit: Eine operative Therapie der Refluxkrankheit bessert die Symptomatik eines gleichzeitig vorliegenden Asthmas, nicht aber die Lungenfunktion und den Medikationsbedarf. Eine Dauermedikation mit $\mathrm{H}_{2}$-Blockern brachte in dieser Studie keine Besserung der Asthmasymptome - möglicherweise wären Protonenpumpenhemmer hier auch eher indiziert gewesen. Die Autoren postulieren eine gegenseitige Beeinflussung von GERD und Asthma, bei der im Laufe der Zeit die durch Aspiration säuregeschädigten Bronchien hypersensitiv gegen eine ganze Reihe von Stimuli werden. Das würde die eingeschränkte Lungenfunktion auch bei fehlender Aspiration erklären.

$b k$

Sontag SJ et al. Asthmatics with gastroesophageal reflux: long term results of a randomized trial of medical and surgical antireflux therapies. Am J Gastroenterol 2003; 98: 987-99 